Cite this: Phys. Chem. Chem. Phys., 2013,

\title{
Spectromicroscopy of pulses transporting alkali metal in a surface reaction $\dagger$
}

15, 8752

Received 12th December 2012,

Accepted 2nd April 2013

DOI: $10.1039 / c 3 c p 44478 c$

www.rsc.org/pccp

\author{
S. Günther, ${ }^{a}$ Hong Liu, $\ddagger^{b}$ T. O. Menteș, ${ }^{c}$ A. Locatellic and R. Imbihl*b
}

\section{Introduction}

Over the past 25 years chemical wave patterns on a catalytic surface have been intensely studied. ${ }^{1-3}$ An enormous variety of patterns have been found ranging from simple target patterns and spiral waves to standing waves, traveling wave fragments, rectangularly shaped target patterns and chemical turbulence. It turned out that nearly all of these patterns can be described using simple activator-inhibitor models already developed in liquid phase chemistry, taking care to add the specific properties introduced by surfaces, i.e. anisotropic surface diffusion and global coupling via the gas phase. A further degree of freedom is generated when an additional component, acting as a "promoter" (or an "inhibitor") for the reaction, is added to the system. This element is mobile, may be more or less reactive but cannot leave the surface, thus becoming part of the catalytic substrate. ${ }^{4,5}$ Such an additional component may typically be an alkali metal (AM). AMs are often added as so-called promoters to heterogeneous catalysts because they enhance the catalytic activity of many transition metal catalysts. ${ }^{9}$

Alkali promoted catalytic surfaces were shown to exhibit the occurrence of Turing-like stationary concentration patterns. ${ }^{4,8,14,15}$

\footnotetext{
${ }^{a}$ TU München, Chemie Department, Lichtenbergstr. 4, D-85748 Garching, Germany

${ }^{b}$ Institut für Physikalische Chemie und Elektrochemie, Leibniz-Universität

Hannover, Callinstr. 3 - 3a, D-30167 Hannover, Germany.

E-mail:imbihl@pci.uni-hannover.de

${ }^{c}$ Sincrotrone Trieste S.C.p.A., S.S. 14, km 163, 5 in AREA Science Park, Basovizza, I-34149 Trieste, Italy

† Electronic supplementary information (ESI) available. See DOI: 10.1039/c3cp44478c

$\ddagger$ Present address: Shanghai Jiao Tong University, Physics Department, Shanghai, China.
}

Such structures are non-equilibrium structures, i.e. they can only be sustained in the presence of an on-going chemical reaction. Unlike the case of true Turing structures, their formation is driven by strong energetic adsorbate-adsorbate interactions and not by the different diffusivities of the reactants. Formally, diffusion is here no longer Fickian and mass transport is controlled by a gradient in the chemical potential. ${ }^{7,8}$ Such patterns are often referred to as the result of a "reactive phase separation". ${ }^{4-6}$ On catalytic surfaces, they have been first observed in the K-promoted $\mathrm{Rh}(110)$ and, later, on a $\mathrm{Rh}(110)$ surface alloyed with $\mathrm{Pd} / \mathrm{Au}$, both systems being exposed to the $\mathrm{O}_{2}+\mathrm{H}_{2}$ reaction. ${ }^{4,8,10-12}$

The formation of stationary $\mathrm{K}+\mathrm{O}$ patterns is, however, not the only novelty occurring using alkali promoted systems but one may also observe pulse transporting potassium as seen in the $\mathrm{NO}+\mathrm{H}_{2}$ reaction on $\mathrm{K} / \mathrm{Rh}(110) \cdot{ }^{13-15}$ When pulses coming from opposite directions collide a substantial accumulation of potassium takes place in the collision area, generating a laterally inhomogeneous $\mathrm{K}$ concentration. After exposing the surface to chemical waves for prolonged time, more complex patterns, such as ordered stationary surface structures ("spider web"), develop, apparently representing a memory effect caused by chemical and/or structural changes of the surface. ${ }^{15}$

The main question that arises for this system concerns the actual mass transport mechanism that is determined by pulse propagation. Chemical waves in the unpromoted system $\mathrm{Rh}(110) / \mathrm{NO}+\mathrm{H}_{2}$ have been investigated in great detail due to a number of intriguing new patterns such as rectangularly shaped target patterns and spiral waves, and traveling wave fragments. ${ }^{16-18}$ These studies led to a mathematical model which could reproduce nearly all of the experiments in great detail. ${ }^{17}$ Since the pulses on the $\mathrm{K}$ promoted surface look very similar to the pulses in the 
unpromoted system one can expect that the basic excitation cycle remains intact in the presence of coadsorbed potassium. What is also known is the strong chemical affinity between oxygen and coadsorbed potassium. This chemical attraction was the basis of the modeling of reactive phase separation in the system $\mathrm{Rh}(110) / \mathrm{K} / \mathrm{O}_{2}+\mathrm{H}_{2} \cdot{ }^{8}$ It turned out, however, that adding attractive energetic interactions between $\mathrm{K}+\mathrm{O}$ to the mathematical model of the unpromoted system could not reproduce the mass transport of potassium by pulses. Only when repulsive interactions between $\mathrm{K}$ and coadsorbed nitrogen were added to the model the simulations yielded pulses which were transporting potassium as observed in the experiment. ${ }^{14}$ A pulse in the system $\mathrm{Rh}(110) / \mathrm{NO}+$ $\mathrm{H}_{2}$ essentially consists of a nitrogen covered surface area, which propagates on a background of the oxygen covered surface. Mathematical modeling demonstrated that, introducing repulsive $\mathrm{K}-\mathrm{N}$ interactions has the consequence that a nitrogen pulse collects $\mathrm{K}$ atoms on the surface pushing them ahead of the pulse similar to a broom sweeping dust. Experimental pulse profiles showed that a substantial $\mathrm{K}$ coverage in fact builds up in front of the pulse thus confirming the proposed mechanism. ${ }^{14}$

Here we report on the experimental study of pulses in the system $\mathrm{Rh}(110) / \mathrm{K} / \mathrm{NO}+\mathrm{H}_{2}$ using "spectromicroscopic" methods in order to identify the different chemical phases and surface structures. Our results represent the basis for formulating the mechanism of mass transport cited above. Already for the unpromoted adsorbate system $\mathrm{Rh}(110) / \mathrm{N}+\mathrm{O}$ a whole zoo of different adsorbate induced reconstructions exist but with potassium again the complexity is strongly increased because a large number of ordered coadsorbate phases are found in the phase diagram for $\mathrm{Rh}(110) / \mathrm{K}+\mathrm{O} \cdot{ }^{19-21}$ By employing synchrotron radiation photoemission electron microscopy in combination with low energy electron microscopy and related methods (SPELEEM), it was possible for us to identify the different phases along a pulse profile. It was shown that a nitrogen adlayer is pushing $\mathrm{K}$ atoms in front of the pulse so that the $\mathrm{K}$ atoms are transported with the pulse. Furthermore, our studies revealed a possible mechanism of how permanent structures on the catalytic surface can be generated through the collision of chemical waves. Such a mechanism is required in order to explain the complex patterns observed in this system. $^{15}$

\section{Experimental}

The experiments were performed at the undulator beamline nanospectroscopy of the Elettra storage ring using the spectroscopic photoemission and low energy electron microscope (SPELEEM III, Elmitec GmbH). The SPELEEM is a special LEEM equipped with a hemispherical band-pass energy filter. By probing the specimen with X-rays, the SPELEEM enables complementary imaging and diffraction techniques to characterize the crystalline, chemical, magnetic and electronic structures of surfaces and interfaces. $^{22}$ Energy and spatial resolution of the microscope approach $0.3 \mathrm{eV}$ and a few tens of nm, respectively. ${ }^{23}$

LEEM uses low energy elastically backscattered electrons to probe the surface structure. It allows for real time imaging with a spatial resolution of about $10 \mathrm{~nm}$. The main contrast mechanism in LEEM is based on electron diffraction. In the bright field mode the primary diffraction beam, or $(0,0)$ beam, is used for imaging, whereas in the dark field mode, secondary diffraction spots generated by overlayers are selected for imaging. Darkfield LEEM enables us to image the lateral extent of the surface phase generating the secondary diffraction beams of interest. Another technique available in a LEEM microscope is mirror electron microscopy (MEM). MEM utilizes very low energy electrons of the order of zero kinetic energy to a few eV that are reflected at the surface without being scattered by the crystal. Contrast arises mainly due to local work function differences and due to variations in the surface topography.

Along with imaging, the SPELEEM allows microprobe diffraction or spectroscopy measurements to be carried out. Microscopic regions of few square microns can be probed by inserting illumination or field limiting apertures in the optical path of the electron beam $(\mu \mathrm{LEED}=$ micro low energy electron diffraction and $\mu$ XPS $=$ micro X-ray photoelectron spectroscopy). Due to spherical and chromatic aberrations of the objective lens, $\mu$ XPS measurements are limited to an area that is slightly larger than the field limiting aperture used in the measurements (diameter $\approx 2 \mu \mathrm{m}$ ).

The $\mathrm{Rh}(111)$ surface sample was cleaned in the preparation chamber of the microscope, by repeated cycles of Ar-ion sputtering at $\sim 750 \mathrm{~K}$ and oxidation at $2 \times 10^{6} \mathrm{mbar}$ and $\sim 1000 \mathrm{~K}$, each step followed by annealing at $1300 \mathrm{~K}$. Potassium was deposited using a SAES getter source, at a deposition rate of $0.001 \mathrm{ML}$ per s. The potassium coverage was calibrated by monitoring the LEED patterns during $\mathrm{K}$ deposition, and the $\mathrm{K}$ flux by recording the time needed to observe K-induced reconstructions of known coverages. At $T=450 \mathrm{~K}$ a saturation coverage of $\theta_{\mathrm{K}}=0.21$ was reached. The $\mathrm{K} 2 \mathrm{p}$ signal of such a phase was used to convert the $\mathrm{K} 2 \mathrm{p}$ intensity into coverage. It is important to note that due to a redistribution of potassium by chemical waves the local $\mathrm{K}$ coverage in an area probed by LEEM, $\mu$ LEED or $\mu$ XPS can be significantly different from the average $\mathrm{K}$ coverage determined at the beginning of the experiment.

The photon energy used for N 1s, K 2p, Rh $3 \mathrm{~d}$ core levels was $460 \mathrm{eV}$; for the $\mathrm{O} 1 \mathrm{~s}$ core level a higher photon energy of $635 \mathrm{eV}$ was chosen. For coverage calibration, the $(1 \times 3)$ low coverage phase of potassium with $\theta_{\mathrm{K}}=0.08$, the $(2 \times 1)$-N with $\theta_{\mathrm{N}}=0.5$ and the $\mathrm{c}(2 \times 8)$-O with $\theta_{\mathrm{O}}=0.75$ were prepared. For determining adsorbate coverages from XPS data the following peak intensity ratios were used, $\mathrm{K} 2 \mathrm{p} / \mathrm{Rh} 3 \mathrm{~d}$ for potassium, $\mathrm{N} \mathrm{1s/Rh} 3 \mathrm{~d}$ for nitrogen and the $\mathrm{O} 1 \mathrm{~s} / \mathrm{Rh} 3 \mathrm{~d}$ ratio for oxygen. If coadsorbates are present in a substantial amount, one overestimates the coverages using these ratios, since the substrate signal ( $\mathrm{Rh} 3 \mathrm{~d}$ ) is damped also by the coadsorbates. We estimate that on surfaces with high coadsorbate coverages, the systematic error amounts to about $20 \%$. The statistical error is of the order of $10 \%$ which includes misalignment. Noise adds to the systematic error.

\section{Results}

\subsection{Lateral composition of the pulses}

(i) Concentration profiles by $\boldsymbol{\mu X P S}$. In the unpromoted system $\mathrm{Rh}(110) / \mathrm{NO}+\mathrm{H}_{2}$ an excitation cycle comprises a simple sequence of only three structural/chemical transformations. 
Starting with the oxygen covered surface as the resting state, the surface is transformed into a nitrogen covered phase as the excited state followed by the backtransformation to the resting state via an $\mathrm{N}+\mathrm{O}$ coadsorbate phase as the refractory state. In short, the excitation cycle is given by: $\mathrm{c}(2 \times 6)-\mathrm{O} \rightarrow(2 \times 1) /$ $(3 \times 1)-\mathrm{N} \rightarrow \mathrm{c}(2 \times 4)-2 \mathrm{O}, \mathrm{N} \rightarrow \mathrm{c}(2 \times 6)-\mathrm{O}$. In a pulse the different phases are spatially separated. The excitation cycle has been demonstrated using $\mu$ XPS, $\mu$ LEED, and via dark-field imaging of pulses in LEEM. ${ }^{17,18,24,25}$ With coadsorbed potassium, the excitation cycle remains basically intact but one should expect that the potassium forms a coadsorbate phase with oxygen in a similar way as it was observed in $\mathrm{Rh}(110) / \mathrm{K} / \mathrm{O}_{2}+\mathrm{H}_{2} \cdot{ }^{20,26}$ Different phases within the pulse train on the K-promoted surface were identified by $\mu$ LEED and $\mu$ XPS under pattern forming reaction conditions.

For instrumental reasons, the total pressure in the LEEM experiments had to be chosen about one to two orders of magnitude lower than in the original PEEM (=photoemission electron microscopy) experiments, i.e. in the $10^{-7}$ mbar range as compared to $10^{-5}$ mbar. Fig. 1 shows a comparison of pulses recorded using PEEM in the $10^{-5}$ mbar range with MEM images of pulses recorded at $10^{-7}$ mbar. $^{14,15}$ In PEEM the nitrogen pulses appear as bright areas propagating on a dark background because the work function (WF) of adsorbed oxygen $\left(\Delta \phi_{\max } \approx 1.1 \mathrm{eV}\right)$ is much higher than the WF of adsorbed nitrogen $\left(\Delta \phi_{\max } \approx 0.3 \mathrm{eV}\right) .^{22}$ The presence of coadsorbed potassium which is known to strongly lower the WF $\left(\Delta \phi_{\max } \approx\right.$ $-4 \mathrm{eV}$ ) only shows up in the collision area of pulses where the accumulation of potassium leads to the formation of a very bright extended surface region. ${ }^{15}$ In the MEM images the contrast is reversed because a higher WF increases the reflectivity of the surface towards incoming electrons. The nitrogen pulses are about $1 \mu \mathrm{m}$ wide propagating with a velocity of ranging between 0.05 and $0.1 \mu \mathrm{m} \mathrm{s}^{-1}$. The white circle in Fig. $1 \mathrm{~b}$ indicates the area from which the photoelectrons are collected in $\mu$ XPS mode. As the pulses pass across the sampled area, one obtains a time dependent variation of the core level intensities. Assuming a constant pulse, velocity, the temporal variations can be transformed into a spatial concentration profile of the pulse train.

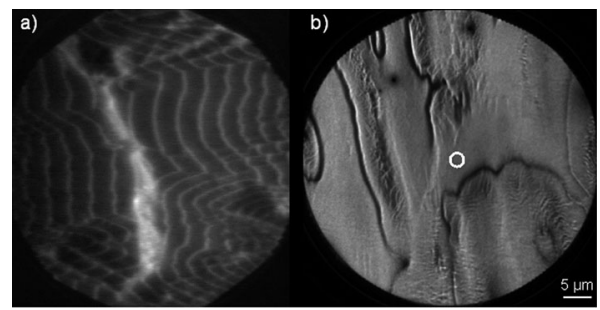

Fig. 1 Comparison of pulses imaged using PEEM (a) and MEM (b). (a) PEEM image showing the enrichment of potassium (bright) in the collision area of pulses. Diameter of the imaged area: $370 \mu \mathrm{m}$. Reaction conditions: $\theta_{\mathrm{K}}=0.08, T=$ $510 \mathrm{~K}, p(\mathrm{NO})=1.5 \times 10^{-6} \mathrm{mbar}, p\left(\mathrm{H}_{2}\right)=1.2 \times 10^{-5} \mathrm{mbar}$ (from ref. 14). (b) MEM image showing pulses propagating with a velocity of $\sim 0.1 \mu \mathrm{m} \mathrm{s}^{-1}$ (MEM: STV = $0.0 \mathrm{~V}, \mathrm{FOV}=50 \mu \mathrm{m})$. The image was taken several hours after deposition of $0.08 \mathrm{ML}$ potassium. Reaction conditions: $\theta_{\mathrm{K}}=0.08, T=469 \mathrm{~K}, \mathrm{p}(\mathrm{NO})=2.5 \times$ $10^{-7}$ mbar, $p\left(\mathrm{H}_{2}\right)=6.7 \times 10^{-7} \mathrm{mbar}$. The white circle indicates the selected area chosen for $\mu$ XPS measurements shown in Fig. 2 and 3.
The spatially resolved photoelectron experiments were conducted on a $\mathrm{Rh}(110)$ surface covered with a total amount of $0.08 \mathrm{ML}$ potassium. Reaction parameters were adjusted so that propagating waves developed on the surface. The MEM image in Fig. 1b displays a pulse train propagating with a pulse velocity of $\sim 0.1 \mu \mathrm{m} \mathrm{s}^{-1}$. The pulses emanate regularly with a period of $\sim 160 \mathrm{~s}$. After positioning the microprobe aperture time dependent $\mu$ XPS measurements were started. About $10 \mathrm{~min}$ ( 4 pulses) after the image in Fig. 1b was acquired the $\mathrm{K} 2 \mathrm{p}+\mathrm{N}$ 1s spectra were recorded as displayed in Fig. 2. The data set was completed by acquiring time resolved $\mathrm{O}$ 1s spectra after the $\mathrm{K} 2 \mathrm{p}$ and $\mathrm{N}$ 1s spectra were recorded (about $30 \mathrm{~min}$ later). The time resolved $\mu$ XPS experiments had to be divided into two subsequently measured data sets, because due to differences in the photoionization cross sections, two different photon energies were selected for the core levels. In order to optimize statistics, essential for real-time monitoring of local photoemission spectra, the experiment was repeated with two different photon energies: $460 \mathrm{eV}$ photons were used for $\mathrm{N} 1 \mathrm{~s}, \mathrm{~K}$ $2 \mathrm{p}$ and $\mathrm{Rh} 3 \mathrm{~d}$ core levels, whereas $\mathrm{O} 1 \mathrm{~s}$, along with $\mathrm{N} 1 \mathrm{~s}$ and $\mathrm{Rh}$ $3 \mathrm{~d}$, was measured at $635 \mathrm{eV}$ photon energy. For a given photon energy, rapid switching of the analyzer energy allows us to establish a reliable phase relationship between different species by repetitively acquiring two spectral regions (e.g. the $\mathrm{N} 1$ s and $\mathrm{K}$ $2 \mathrm{p}$ spectra). In this way, the lower photon energy was utilized to correlate $\mathrm{N} 1 \mathrm{~s}$ and $\mathrm{K} 2 \mathrm{p}$ signals, which was complemented by the high photon energy measurements used to obtain the correlation between $\mathrm{N}$ 1s and $\mathrm{O} 1 \mathrm{~s}$.

The results of the $\mu$ XPS measurements are displayed in Fig. 2 and 3 showing the time-dependent variation of the $\mathrm{K}, \mathrm{N}$, and $\mathrm{O}$ coverages together with the spectral information. Sequences of subsequently acquired spectra are displayed in the so-called waterfall plots, which are two-dimensional representations of the data. The electron energy is given along the horizontal axis, the vertical axis corresponds to the time scale, and the spectral intensity is coded as the brightness of the image. Each horizontal cut through such an image yields the photoelectron spectrum at that particular time, as indicated below the $\mathrm{K} 2 \mathrm{p}$ and $\mathrm{N} 1 \mathrm{~s}$ waterfall panels in Fig. 2 .

The oxygen trace in Fig. 3 reflecting the passage of the pulse train shows that the amount of oxygen varies from a rather high coverage of $\theta_{\mathrm{O}}=0.8$ to an oxygen concentration below the detection limit $(<0.1 \mathrm{ML})$. This shows that a fully oxygen covered surface is completely reduced by a nitrogen pulse followed by the return to a fully oxygen covered surface. The O 1s intensity displays a rather broad peak at around $529.7 \mathrm{eV}$ binding energy, which most likely consists of several components in accordance with high resolution XPS data obtained from $\mathrm{Rh}(110) / \mathrm{K}+\mathrm{O}^{26}$

In contrast to oxygen, the nitrogen signal never completely vanishes but undergoes a modulation from $\theta_{\mathrm{N}}=0.34$ at maximum to $\theta_{\mathrm{N}}=0.15$ at minimum within a pulse. Remarkably, the $\mathrm{N}$ 1s spectra in Fig. 2 and 3 clearly reveal the presence of two components separated by about $0.3 \mathrm{eV}$. The variations of each of the $\mathrm{N} 1 \mathrm{~s}$ components alone and of the total nitrogen coverage are plotted in Fig. 3. The diagram shows that the component at 

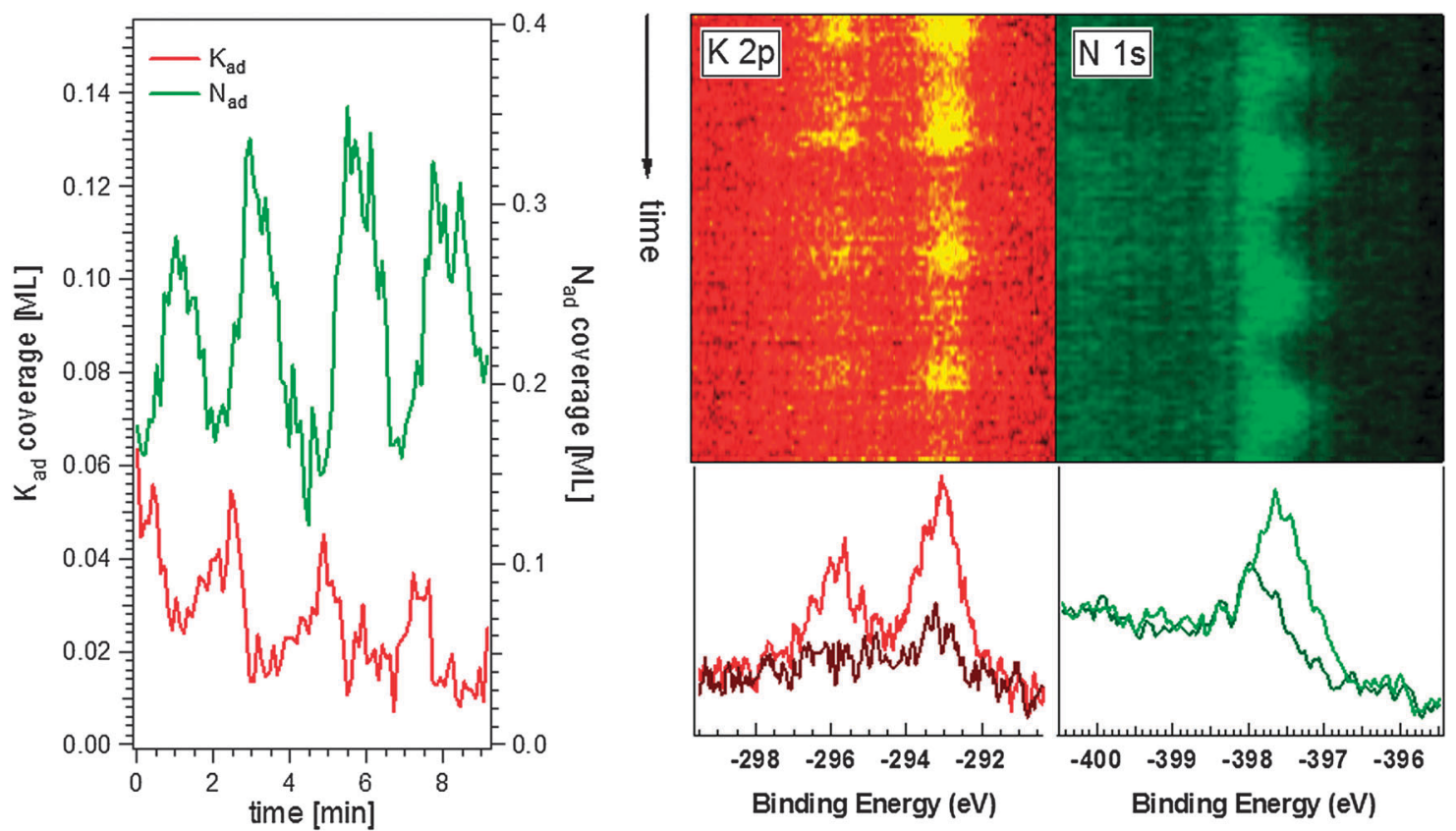

Fig. 2 Variation of the potassium and nitrogen coverage in a pulse train showing a progressive loss of the local K coverage with time. The data were measured with $\mu$ XPS revealing the presence of two different nitrogen species. Left panel: time dependent variation of the $K$ coverage measured as pulses pass the area probed by $\mu$ XPS. Right panel: top: K $2 p$ and N $1 s$ core level spectra as a function of time displayed in a waterfall plot. Spectra corresponding to a horizontal cut through both waterfall plots were recorded in a row (see text). Bottom: two spectra selected from the above data sets at maximum and minimum intensity of $\mathrm{N} 1 \mathrm{~s}$ and $\mathrm{K} 2 \mathrm{p}$. Reaction conditions: $\theta_{\mathrm{K}}=0.08, T=469 \mathrm{~K}, p(\mathrm{NO})=2.5 \times 10^{-7} \mathrm{mbar}, p\left(\mathrm{H}_{2}\right)=6.7 \times 10^{-7} \mathrm{mbar}$.
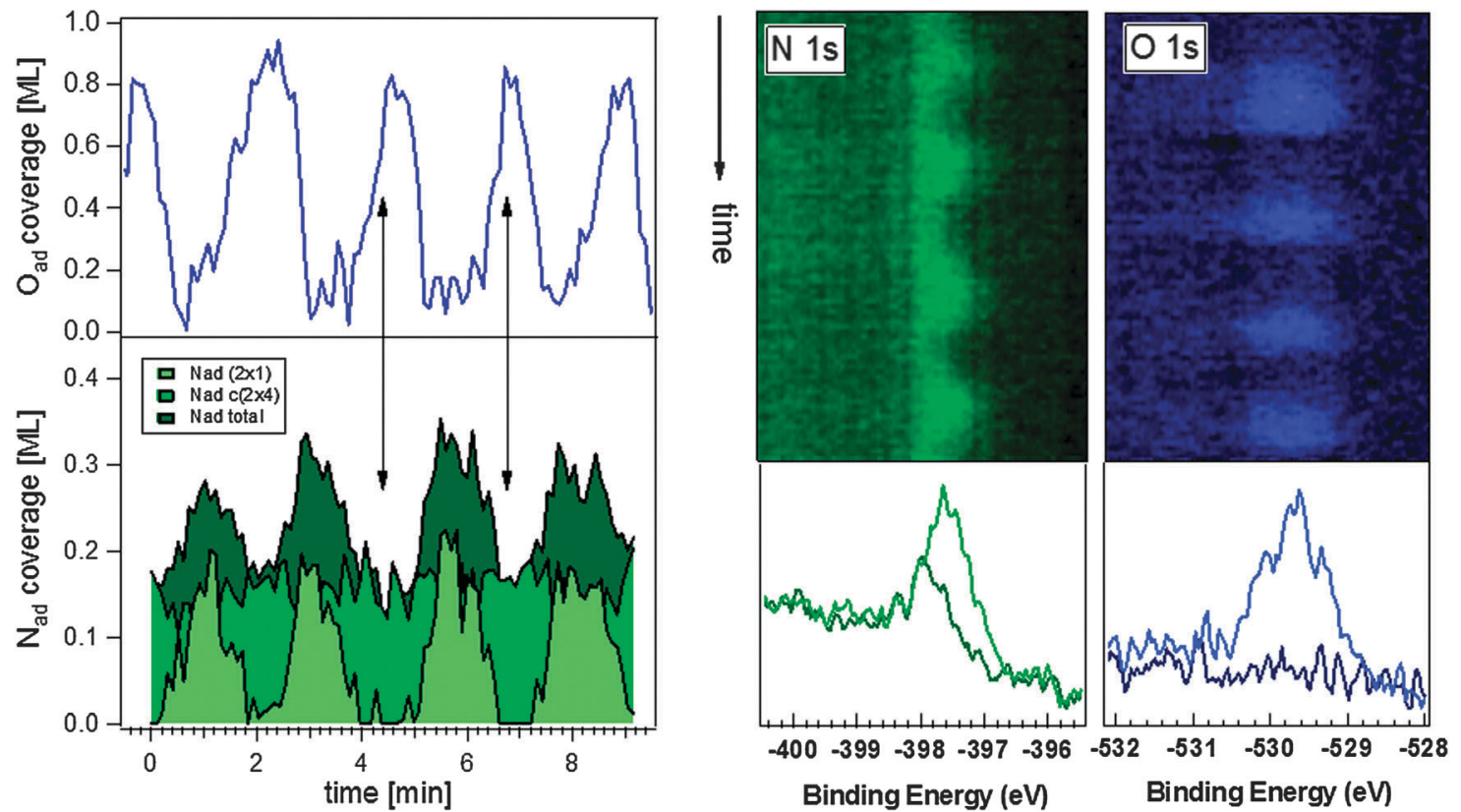

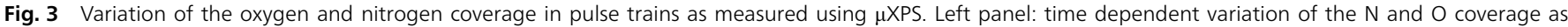

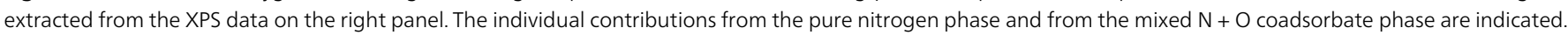

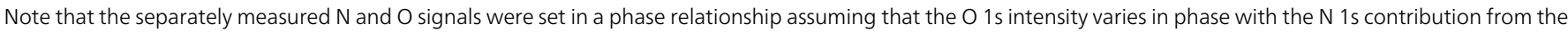

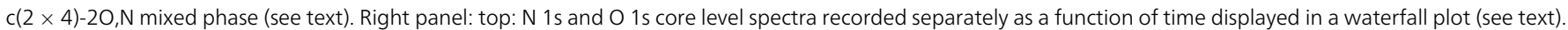

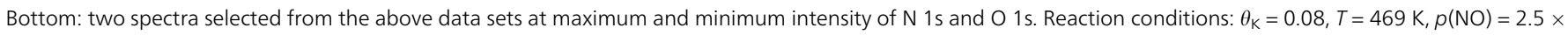
$10^{-7}$ mbar, $p\left(\mathrm{H}_{2}\right)=6.7 \times 10^{-7}$ mbar.

lower binding energy periodically vanishes and reappears in a pulse train, whereas the high binding energy component exhibits a much weaker intensity modulation. The two $\mathrm{N} 1 \mathrm{~s}$ components can be assigned to two different nitrogen species: 
chemisorbed nitrogen in the $(2 \times 1)-\mathrm{N}$ phase and nitrogen coadsorbed with oxygen in the mixed $\mathrm{c}(2 \times 4)-2 \mathrm{O}, \mathrm{N}$ phase. ${ }^{18,24,25}$ In the literature, the $\mathrm{N}$ 1s binding energy for the $(3 \times 1)-\mathrm{N}$ phase on $\mathrm{Rh}(110)$ (similar to the $(2 \times 1)-\mathrm{N}$ phase) is reported to be $397.3 \mathrm{eV}$ and the one corresponding to the mixed $\mathrm{c}(2 \times 4)$-NO coadsorbate phase equals $397.6 \mathrm{eV} .{ }^{27}$ The separation of $0.3 \mathrm{eV}$ is exactly the one we observe in the experiment. Note that this assignment correlates the $\mathrm{O} 1 \mathrm{~s}$ and the $\mathrm{N}$ 1s modulation in time as indicated in Fig. 3. The assignment of the nitrogen species is further supported by the time-dependent LEED measurements that will be summarized in the following section.

The results of the $\mathrm{K} 2 \mathrm{p}$ measurements are displayed together with the N 1s data in Fig. 2. Similar to the oxygen and nitrogen signals the potassium coverage exhibits a periodic variation. However, in contrast to the other constituents, the potassium signal on average shows a steady decay. This indicates that the average potassium coverage decreases strongly during a few cycles. The decaying $\mathrm{K}$ signal can be explained by each pulse carrying away a certain amount of potassium and therefore progressively reducing the local K coverage. Quantitatively, the average decrease of potassium coverage for the first few pulses is about 0.01 to $0.02 \mathrm{ML}$ per pulse, as seen in Fig. 2 . The locally observed loss of $\mathrm{K}$ requires a mass balance in which the potassium swept by the pulses has to accumulate in other areas. Indeed, as we will show in the following sections, regions exist where potassium accumulates.

Essential information for the mechanistic interpretation of the mass transport is the phase relationship between the coverage of $\mathrm{K}$ and the coverages of the other adsorbates in a pulse. In the experiment displayed in Fig. 2 rapid switching between $\mathrm{K} 2 \mathrm{p}$ and $\mathrm{N}$ 1s spectrum acquisition allowed for determining this phase relationship. As demonstrated by the coverage plot of Fig. 2 the K 2p maxima occur sharply before the $\mathrm{N}$ 1s maxima. In terms of spatial coordinates, the pulse propagation direction is from right to left on the left panel in Fig. 2. This means that the nitrogen of the $(2 \times 1)-\mathrm{N}$ in a pulse is pushing the potassium. In other words, the nitrogen adlayer of a pulse acts like a broom sweeping up dust. Thus a large potassium concentration builds up in front of the nitrogen pulse.

Spectra of the $\mathrm{K} 2 \mathrm{p}$ region recorded at the maximum and minimum of the $\mathrm{K}$ coverage are displayed in the lower part of Fig. 2. The $\mathrm{K} 2 \mathrm{p}_{3 / 2}$ peak exhibits a binding energy (BE) of $293.5 \mathrm{eV}$. For comparison, for the pure metallic system $\mathrm{Rh}(110) / \mathrm{K} \mathrm{a}$ $\mathrm{BE}$ of $293.6 \mathrm{eV}$ was reported for $\mathrm{K} 2 \mathrm{p}_{3 / 2}$ whereas the corresponding $\mathrm{BE}$ for the coadsorbate system $\mathrm{Rh}(110) / \mathrm{K}+\mathrm{O}$ is $292.9 \mathrm{eV} .^{26}$ One has to conclude that the transported potassium is in a metallic state rather than being coadsorbed with oxygen. The rather broad appearance of the $\mathrm{K} 2 \mathrm{p}_{3 / 2}$ peaks indicates the coexistence of more than one species.

(ii) Ordered overlayers in the pulses. The temporal variations in the surface structure are obtained using LEEM and $\mu$ LEED measurements displayed in Fig. 4. The top image corresponds to the passage of a nitrogen pulse across the area probed by LEED. An intense $(2 \times 1)$-N pattern is seen together with faint $\mathrm{c}(2 \times 4)$ spots. A dark field LEEM image taken with the $(1 / 2,0)$ beam of the $(2 \times 1)-\mathrm{N}$ highlights the propagating pulses as demonstrated in the upper right diagram of Fig. 4. A short time later, the $(2 \times 1)$ - $\mathrm{N}$ spots are already quite weak whereas the $\mathrm{c}(2 \times 4)$ pattern has increased its intensity (second LEED pattern from top). In the last pattern of the sequence, taken well after passage of the nitrogen pulse, only an intense $c(2 \times 4)$ remains visible. The diagram on the right side of Fig. 4 displays the intensity variation of two diffraction beams, the $(0,1 / 2)$ and the $(1 / 2,1 / 4)$ beam, representing the two patterns. The $(2 \times 1)$ intensity exhibits the spike-like appearance characteristic for nitrogen pulses. The $\mathrm{c}(2 \times 4)$ intensity shows a complementary behavior to the $(2 \times 1)-\mathrm{N}$. Unlike the unpromoted system, the $\mathrm{c}(2 \times 4)$ never completely vanishes but always persists with a certain intensity. ${ }^{18,24,25}$ One also notes that the $(0,1 / 2)$ beam whose intensity is strongly related to the $(1 \times 2)$ "missing row" reconstruction never completely vanishes.

The pulses propagate only on areas with low $\mathrm{K}$ coverage but not on $\mathrm{K}$ enriched areas. This behavior is demonstrated in the middle image of the right column in Fig. 4 (see also movie in ESI, $†$ speed-up factor $=50$ ). The middle image was constructed by adding up 70 subsequently recorded dark field images of the $(2 \times 1)-\mathrm{N}$. The area in which pulses propagate appears bright whereas a dark grey contrast indicates regions where pulse propagation is suppressed. A comparison with the MEM image (bottom image) shows that the area where the pulse propagation is suppressed exhibits a lower work function which appears dark in MEM. This dark area reflects a strong $\mathrm{K}$ enrichment.

Neither the $\mathrm{c}(2 \times 6)-\mathrm{O}$ nor any other pure oxygen phase of $\mathrm{Rh}(110) / \mathrm{O}_{2}$ were seen in the experiments. Since the oxygen coverage after passage of a nitrogen pulse with $\theta_{\mathrm{O}} \approx 0.8$ is very high (Fig. 2 and 3), it is evident that the oxygen has to be present as a coadsorbate phase together with potassium. Both, the pure alkali metal phases $\mathrm{Rh}(110) / \mathrm{K}$ as well as the coadsorption phases $\mathrm{Rh}(110) / \mathrm{K}+\mathrm{O}$ were studied in detailed LEED, STM and XPS experiments. ${ }^{20,26}$ A whole variety of different reconstruction phases exists which are all based on the "missing row" principle. The net effect of coadsorbed potassium is that (i) the surface can accommodate much higher oxygen coverages than without AM, i.e. up to $\theta_{\mathrm{O}} \approx 1.5$ as compared to $\theta_{\mathrm{O}} \approx 1$ of $\mathrm{Rh}(110) / \mathrm{O}_{2}$ and that (ii) the reactivity of oxygen towards $\mathrm{H}_{2}$ is drastically reduced at higher $\mathrm{K}$ coverages. Mechanistically, the latter effect was attributed to a lowering of the reactive sticking coefficient of $\mathrm{H}_{2}$ by coadsorbed potassium. ${ }^{26}$

In the patterns observed with $\mu$ LEED no extra spots were detected besides the $(2 \times 1)-\mathrm{N}$ and $\mathrm{c}(2 \times 4)$ spots which could be related directly to any of the numerous ordered $\mathrm{K}+\mathrm{O}$ coadsorption phases on $\mathrm{Rh}(110)$. A clue where the potassium is located is obtained from the observation that the $c(2 \times 4)$ intensity in Fig. 4 never vanishes as opposed to the behavior of the $c(2 \times 4)$ $2 \mathrm{O}, \mathrm{N}$ in the unpromoted system. At intermediate $\mathrm{K}$ coverages, around $\theta_{\mathrm{K}} \approx 0.12$, a $\mathrm{c}(2 \times 4)$ phase appears in the $\mathrm{K}+\mathrm{O}$ coadsorption system after dosing about 1 Langmuir $\mathrm{O}_{2}$ onto the $\mathrm{K}$ covered surface corresponding to an estimated oxygen coverage of $\approx 0.5 .^{20}$ Apparently, the $\mathrm{c}(2 \times 4)$ we see in $\mu$ LEED contains two contributions, one from the $\mathrm{O}+\mathrm{N}$ coadsorption system, the $\mathrm{c}(2 \times 4)-2 \mathrm{O}, \mathrm{N}$ and a second one from the $\mathrm{K}+\mathrm{O}$ coadsorption system, both exhibiting a $(1 \times 2)$ missing row reconstruction. 

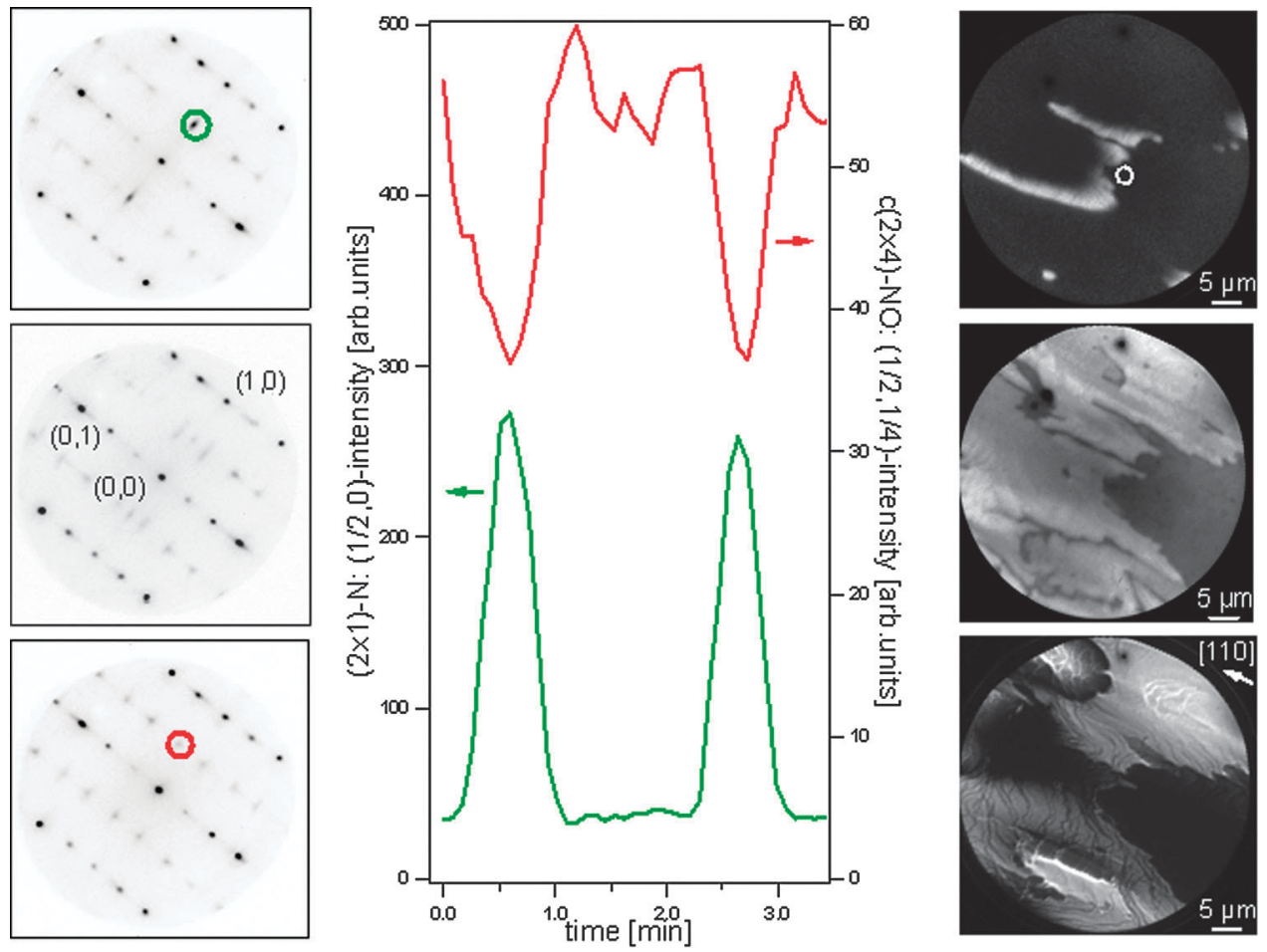

Fig. 4 Ordered adsorbate phases in a pulse train as measured using $\mu$ LEED. Left panel: $\mu$-LEED patterns ( $40 \mathrm{eV})$ subsequently acquired (from top to bottom) during propagation of a pulse train. Top: $(2 \times 1)$ pattern in coexistence with weak $c(2 \times 4)$ spots. Green circle indicates the $(2 \times 1)$ spot used for the plot in the center panel. Middle: decay of $(2 \times 1)$ intensity and development of the $c(2 \times 4)$ phase with disorder along the [110]-direction. Bottom: well developed $c(2 \times 4)$ pattern; red circle indicates the $\mathrm{c}(2 \times 4)$ spot used for the plot in the center panel. Center panel: temporal evolution of $(1 / 2,0)$ - and $(1 / 2,1 / 4)$-spot intensity reflecting the abundance of the $(2 \times 1)$ and $(2 \times 4)$ phase during propagation of a pulse train (spots indicated by a green and a red circle in the left panel). Right panels: LEEM/MEM images showing the contributions of different phases to chemical waves. Field of view: $50 \mu \mathrm{m}$. Top: dark field (DF) LEEM image $(35 \mathrm{eV}$, (1/2,0)-spot) of a traveling pulse demonstrating the contribution of the $(2 \times 1)-\mathrm{N}$ phase (see movie in ESI, † speed-up factor $=50)$. Middle: superposition of 70 subsequently acquired DF images during the passage of a pulse train (see text). Bottom: MEM image ( $\mathrm{STV}=1.0 \mathrm{~V}$ ) showing the $\mathrm{K}$-rich zone as a dark area. Reaction conditions: $\theta_{\mathrm{K}}=0.08, T=469 \mathrm{~K}, p(\mathrm{NO})=$ $2.5 \times 10^{-7} \mathrm{mbar}, \mathrm{p}\left(\mathrm{H}_{2}\right)=4.7 \times 10^{-7} \mathrm{mbar}$.

Evidently, the $(2 \times 1)$ - $\mathrm{N}$ is transformed into the $\mathrm{c}(2 \times 4)-2 \mathrm{O}$, $\mathrm{N}$ as in the unpromoted system but then the $\mathrm{c}(2 \times 4)-2 \mathrm{O}, \mathrm{N}$ is replaced by a $\mathrm{c}(2 \times 4)$ of the $\mathrm{K}+\mathrm{O}$ coadsorption system which has the consequence that the $\mathrm{c}(2 \times 4)$ intensity remains high.

With rising oxygen coverage the $\mathrm{c}(2 \times 4)$ transforms into a $c(6 \times 4)$; in LEED this transformation is indicated by a continuous splitting of the $(1 / 2,1 / 4)$ spots. $^{20}$ In the $\mu$ LEED pattern in Fig. 4 we see a broadening of the $(1 / 2,1 / 4)$ spots but at the end no distinct $c(6 \times 4)$ pattern can be identified. This indicates a substantial amount of disorder and in fact a considerable amount of potassium may not be bound in a well ordered $\mathrm{c}(2 \times 4)$ phase but may exist as a disordered phase on $1 \times 2$ reconstructed regions of the surface.

According to $\mu$ XPS (Fig. 2) the nitrogen coverage never goes to zero. However, this should be the case if the $\mathrm{K}+\mathrm{O}$-coadsorbate phase is part of the excitation cycle (see below). This apparent contradiction can be resolved if we assume that the $\mathrm{K}+\mathrm{O}$ coadsorbate phase is spatially confined to an area smaller than the probing spot of $\sim 2 \mu \mathrm{m}$. A too large probing window compared to the dimensions of a pulse could thus explain the non-vanishing $\mathrm{N}$-intensity.

Rather than forming a mixed phase with three constituents, $\mathrm{K}, \mathrm{N}$, and $\mathrm{O}$, a separation of the adsorbates into a $\mathrm{K}+\mathrm{O}$ and $\mathrm{a}$
$\mathrm{N}+\mathrm{O}$ phase, both with a $\mathrm{c}(2 \times 4)$ registry, is likely. The appearance of rather broad $c(2 \times 4)$ LEED spots supports this assumption, because if we take the broad spots as an indication of small adsorbate islands then the average island size will be below the lateral resolution of the $\mu$-probe experiment. The latter condition is required in order to be consistent with the experimental data.

In experiments performed at about $50 \mathrm{~K}$ higher temperature a $\mathrm{c}(6 \times 4)$ phase attributed to the $\mathrm{K}+\mathrm{O}$ coadsorbate develops as demonstrated in Fig. 5. The pulses under these conditions propagate with a speed of $0.22 \mu \mathrm{m} \mathrm{s}^{-1}$. In the $\mu$ LEED pattern the splitting of the $(1 / 2,1 / 4)$ spots into $(1 / 3,1 / 4)$ and $(2 / 3,1 / 4)$ spots is clearly seen. A plot of the integrated spot intensity of the various ordered overlayers $v s$. the time in Fig. 5 shows a dominant $(2 \times 1)$ - $\mathrm{N}$ pattern which persists over a long time, i.e. the pulse is rather broad. The development of the $(2 \times 1)-\mathrm{N}$ is preceded by short spike-like appearance of the $(3 \times 1)$-N whose coverage $\theta_{\mathrm{N}}=1 / 3$ is smaller than the $\theta_{\mathrm{N}}=0.5$ of the $(2 \times 1)-\mathrm{N}$. Similar observations were made in the unpromoted system. ${ }^{18}$ The disappearance of the $(2 \times 1)-\mathrm{N}$ is connected with formation of the $\mathrm{c}(2 \times 4)$ structure representing the ordered mixed coadsorbate phases of $\mathrm{N}+\mathrm{O}$ and of $\mathrm{O}+\mathrm{K}$. The observation of a $c(6 \times 4)$ pattern indicates the formation of the $\mathrm{K}+\mathrm{O}$ coadsorbate. 

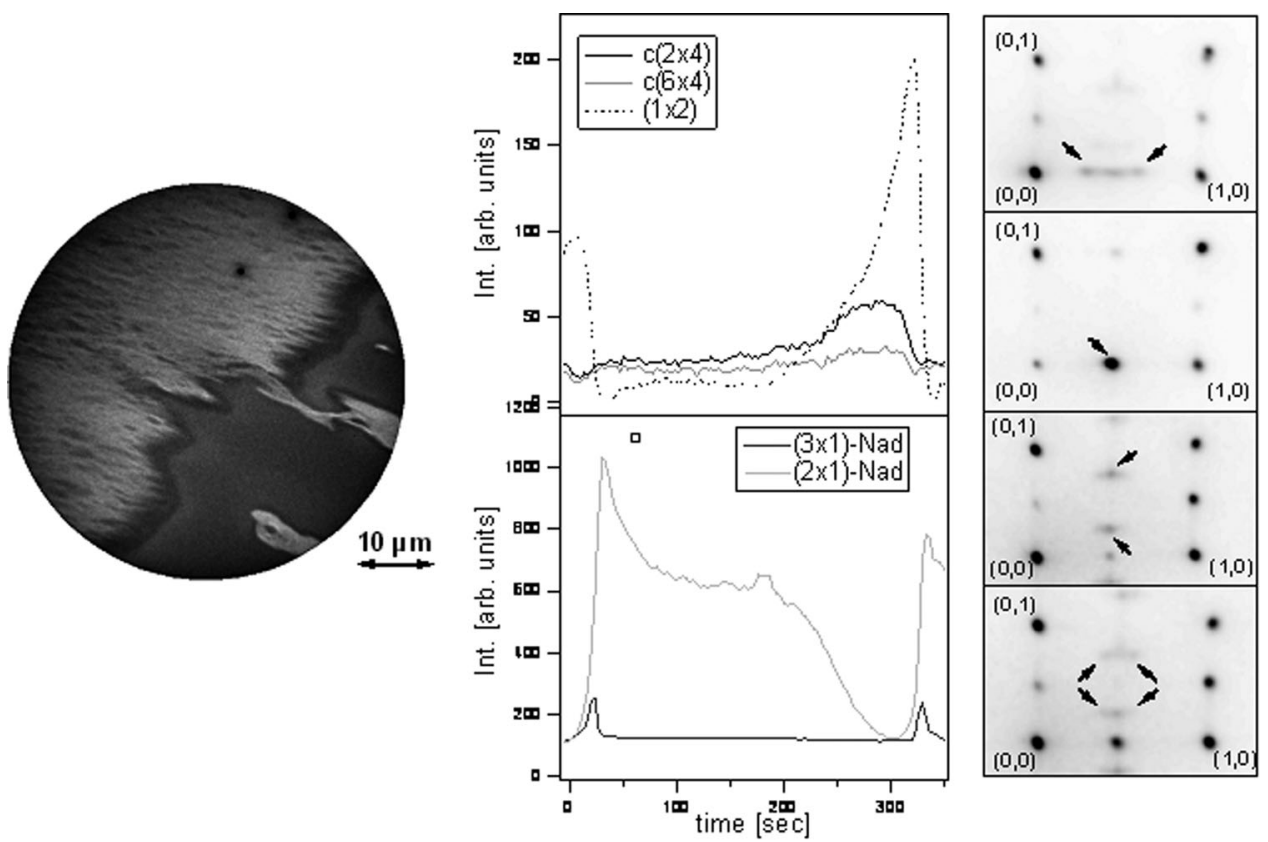

Fig. 5 Appearance of the $c(6 \times 4) \mathrm{K}+\mathrm{O}$ coadsorbate phase in a pulse train as measured using $\mu$ LEED. Left panel: MEM image $(S T V=2.04 \mathrm{~V}$, FOV $=50 \mu \mathrm{m})$ showing a broad pulse propagating from right to left. Pulse velocity $=0.22 \mu \mathrm{m} \mathrm{s}^{-1}$. Center panel: temporal evolution of the various ordered overlayers in the pulse as measured using $\mu$-LEED $(43 \mathrm{eV})$ as measured by spot intensities of $c(2 \times 4):(1 / 2,1 / 4) / c(2 \times 6):(1 / 3,1 / 4) /(1 \times 2):(0,1 / 2) /(2 \times 1)-\mathrm{N}$ : $(1 / 2,0) /(3 \times 1)$-N: $(1 / 3,0)$. Note the different scales and the different offsets for the various overlayers. Right panel: sequence of $\mu$-LEED patterns $(40 \mathrm{eV})$ showing the presence of different ordered overlayers in a pulse. 1 st: $(2 \times 1)-\mathrm{N}+(3 \times 1)-\mathrm{N}, 2$ nd: $(2 \times 1)-\mathrm{N}, 3 \mathrm{rd}: \mathrm{c}(2 \times 4)$, 4th: $\mathrm{c}(6 \times 4)+\mathrm{c}(2 \times 4)$. Reaction conditions: $\theta_{\mathrm{K}}=0.08, T \approx 520 \mathrm{~K}, p(\mathrm{NO})=2.0 \times 10^{-7} \mathrm{mbar}, p\left(\mathrm{H}_{2}\right)=$ $2.4 \times 10^{-7} \mathrm{mbar}$

Simultaneously, the intensity of the $(1 \times 2)$ reconstruction monitored here by the $(0,1 / 2)$-beam strongly grows. While the sequence of ordered layers is well reflected in the middle panel of Fig. 5, the exact phase relationship between the intensities of the different overlayer beams should not be considered, because this relationship is only exact within $10 \mathrm{~s}$. The reason is that different regions in reciprocal space do not exactly stem from the same probing area in real space as it would be the case ideally.

\subsection{Potassium accumulation by pulse collision}

In the MEM/LEEM experiments the accumulation of potassium is more difficult to follow than in the PEEM studies, because at lower total pressure reaction conditions leading to chemical pattern formation are more difficult to adjust due to reduced dynamics. ${ }^{15}$ The principle effect of an accumulation of potassium through the collision of pulses can nevertheless be demonstrated as shown by the MEM/ $/$ XPS experiment in Fig. 6. The MEM image on top displays a snapshot of the pulse train; the encircled region marks the area over which the photoelectrons are collected. The anisotropy of the moving fronts which can be already seen in Fig. 4 is caused by diffusional anisotropy with the long axis of the elliptically deformed patterns being given by the orientation of the "[110]-troughs" of Rh(110). Over the next $30 \mathrm{~min}$ a number of pulses collide in the area sampled by $\mu$ XPS (see white circle in Fig. 6). The time dependent photoemission signals, also displayed in Fig. 6, show that the nitrogen pulses slowly diminish and cease after about 15 minutes. On the other hand, the potassium signal steadily increases, indicating $\mathrm{K}$ accumulation.

In the bottom MEM image, which illustrates the final situation, the accumulated potassium appears as a large dark area, in which the brightness reflects the lowering of the WF by potassium. In this dark area pulse propagation is suppressed, which has already been demonstrated in Fig. 4 . Note that, since the photoemission data in Fig. 6 are not calibrated no absolute coverages can be given. The suppression of pulse propagation is not a principle effect but results simply from a lowering of the $\mathrm{H}_{2}$ sticking coefficient by increasing potassium coverage. ${ }^{26}$ This causes the reaction conditions to drift outside the excitable parameter range. As will be shown below, by increasing $p\left(\mathrm{H}_{2}\right)$ pulse propagation can be reestablished.

\subsection{Evaporation of potassium under pattern forming conditions}

In a series of experiments different amounts of potassium were deposited onto $\mathrm{Rh}(110)$ under reaction conditions while simultaneously the effect of a rising $\mathrm{K}$ coverage on the patterns was studied using MEM. The starting point in each case was the K-free $\mathrm{Rh}(110)$ surface. In separate experiments altogether three $\mathrm{K}$ coverages were deposited, $\theta_{\mathrm{K}}=0.03,0.08$, and 0.12 corresponding to evaporation times of $30 \mathrm{~s}, 70 \mathrm{~s}$, and $110 \mathrm{~s}$, respectively.

The first frame in Fig. 7a shows a target pattern on the K-free surface in the moment of switching on the K-evaporator. After a delay of 10-20 s caused by the time it takes to heat up the 

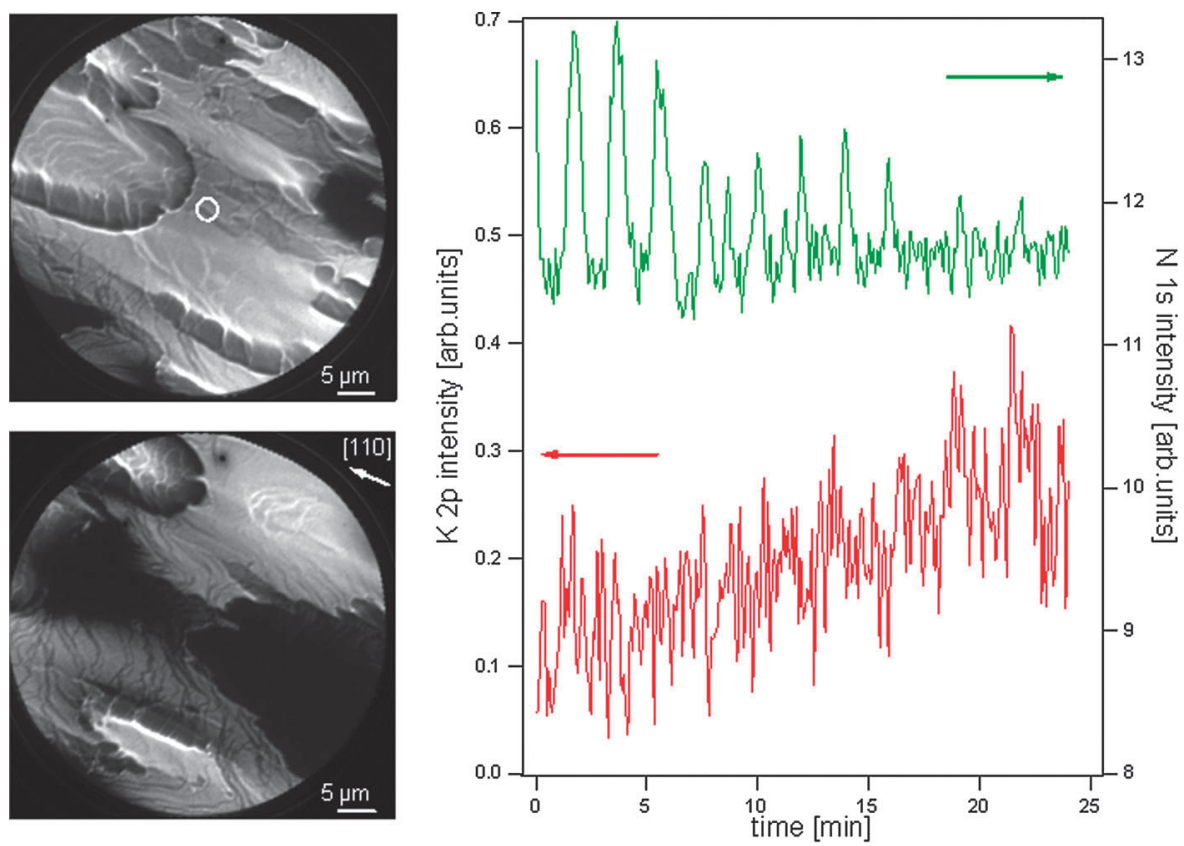

Fig. 6 Large scale enrichment of potassium caused by collision of pulses. Left panel: MEM images (STV $1.0 \mathrm{eV}$, FOV $\sim 50 \mu \mathrm{m})$ showing pulse propagation 3 min before (top graph) and $40 \mathrm{~min}$ after (bottom graph) the $\mu$-XPS experiment (right panel) performed on an area which was greatly $\mathrm{K}$ depleted at $t=0 \mathrm{~min}$. The bottom image is identical to the one shown in Fig. 4 (right panel, bottom). Right panel: local $\mathrm{N} 1 \mathrm{~s}$ and $\mathrm{K} 2 \mathrm{p}$ core level intensity vs. time showing $\mathrm{K}$ enrichment in the area indicated by the marked circle in the MEM image of the left upper panel. Reaction conditions: $\theta_{\mathrm{K}}=0.08, T=469 \mathrm{~K}, p(\mathrm{NO})=2.5 \times 10^{-7} \mathrm{mbar}, p\left(\mathrm{H}_{2}\right)=4.7 \times 10^{-7} \mathrm{mbar}$.

evaporation source $0.03 \mathrm{ML}$ potassium were deposited over a period of $\approx 30 \mathrm{~s}$. One observes initially a darkening of the pulse as well as of the surrounding area reflecting the WF decrease of both by adsorbing potassium. At one point (40 s) the nitrogen pulse almost becomes indistinguishable from the surrounding area. Apparently, the addition of a small amount of potassium does not suppress the excitability of the medium; also the anisotropy of the patterns is not notably changed through the addition of potassium. On such a weakly promoted surface the accumulation of potassium through the collision of pulses shows up very clearly as demonstrated by a series of MEM images in Fig. 7b. Here, the K-transport and enrichment in the collision area result in the local lowering of the work function (WF), which is imaged in MEM as a pronounced darkening. Unfortunately, the relation of the grey levels in MEM images to differences in the $\mathrm{K}$ coverage is generally problematic for two reasons. Firstly, the reflectivity of the surface is not a linear function of the WF but exhibits a threshold behavior, i.e. below a certain threshold any modification of the WF will not have a detectable influence on the brightness. The second problem is the non-linear behavior of the $\mathrm{WF}$ in the $\mathrm{K}+\mathrm{O}$ coadsorption system with respect to coverage. ${ }^{15}$ Exposing a potassium covered surface to a high dose of oxygen $(136 \mathrm{~L})$ one finds a small effect of potassium up to $\theta_{\mathrm{K}} \approx 0.05$ whereas addition of potassium in a range from $\theta_{\mathrm{K}}=0.05$ to $\theta_{\mathrm{K}}=0.12$ causes a drastic WF decrease by $1.7 \mathrm{eV}$. Further increasing $\theta_{\mathrm{K}}$ leads only to little variation of the WF.

Although no simple correlation between the absolute grey level in MEM images and the K coverage can be made the effect of $\mathrm{K}$ codeposition on pattern formation can be demonstrated very clearly applying an adaptive grey scale. In this case, only relative changes in electron reflectivity within each image (caused by local changes in the WF) provide the contrast. During recording a sequence of MEM images the center of the grey scale was continuously adapted to the change in reflectivity by changing the start voltage from image to image. In this way one compensates for the global WF change keeping the electron reflectivity almost constant. This approach was used in the experiment displayed in Fig. 8. Alternatively, an adaptive grey scale can simply be obtained by continuously rescaling the grey levels to the minimum and maximum level in each image. A mixture of both techniques can be applied if the WF varies over a large range within a single sequence of images. This latter procedure was used during the acquisition of the images displayed in Fig. 9.

By resorting to an adaptive grey scale the more drastic effects on the patterns connected with larger amounts of deposited potassium can still be imaged as demonstrated in Fig. 8. In this case up to $\theta_{\mathrm{K}}=0.08$ were evaporated onto $\mathrm{Rh}(110)$. In this sequence a contrast reversal between the imaged pulses and the surrounding medium takes place between 20 and 30 s exposure time. Reduction fronts which prior to $\mathrm{K}$ codeposition appear darker than the surrounding medium (indicating a low WF in the chemical wave) turn at higher $K$ coverage brighter than the surrounding medium (indicating that now the surrounding medium has a lower WF than the pulse). This peculiar contrast reversal is a result of the abovementioned non-linear change of the $\mathrm{WF}$ in the $\mathrm{K}+\mathrm{O}$ coadsorbate with rising $\mathrm{K}$ coverage. Beyond $60 \mathrm{~s}$ exposure time pulse propagation is suppressed and the wave patterns start to decay (see images at $80 \mathrm{~s}, 100 \mathrm{~s}$ and $200 \mathrm{~s}$ ). 
a)
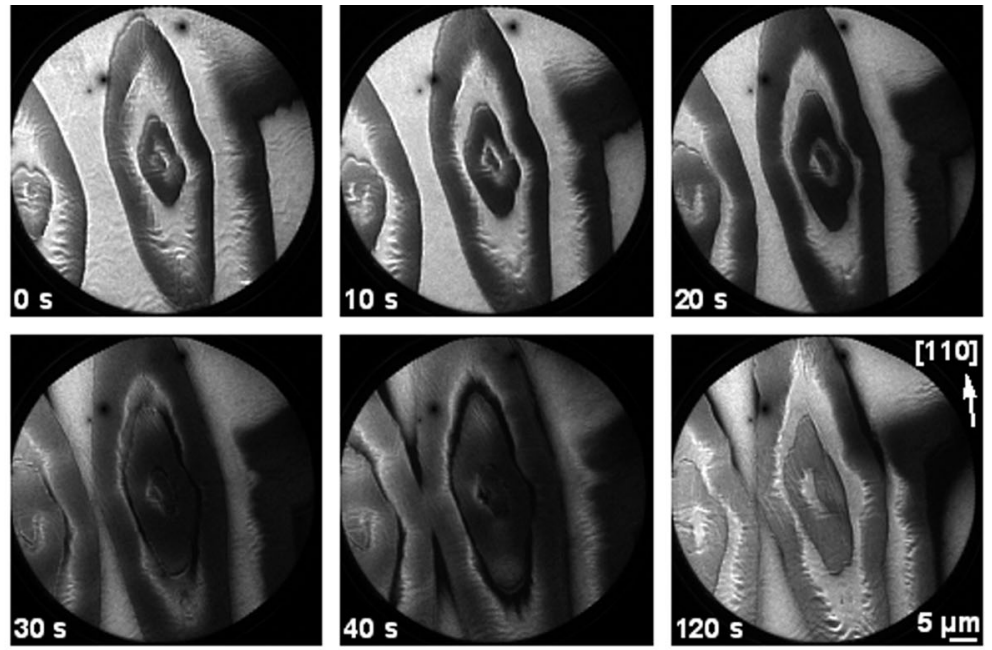

b)
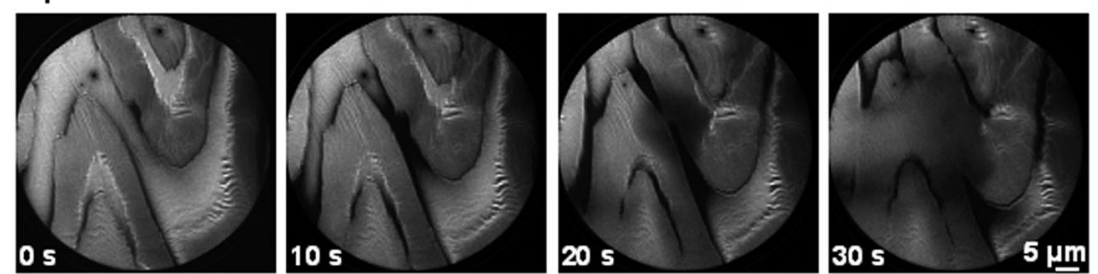

Fig. 7 Effect of in situ evaporation of $0.03 \mathrm{ML}$ potassium on chemical wave patterns starting from a K-free surface. (a) Sequence of MEM images (STV $=1.25 \mathrm{~V}$, FOV $=$ $50 \mu \mathrm{m}$, fixed grey scale) acquired during evaporation of potassium (30 s evaporation time) onto a target pattern. Start of the evaporation between 10 and $20 \mathrm{~s}$. Coadsorbed K shows up as the darkened area reflecting the local work function decrease. (b) Sequence of MEM images showing pulse collision in detail. The images were acquired shortly after the MEM images in (a). Reaction conditions: $T=473 \mathrm{~K}, p(\mathrm{NO})=2.5 \times 10^{-7} \mathrm{mbar}, p\left(\mathrm{H}_{2}\right)=7.0 \times 10^{-7} \mathrm{mbar}$ (see also movies in ESI, $\dagger$ speed-up factor $=100$ for $a, 17$ for $b$ ).
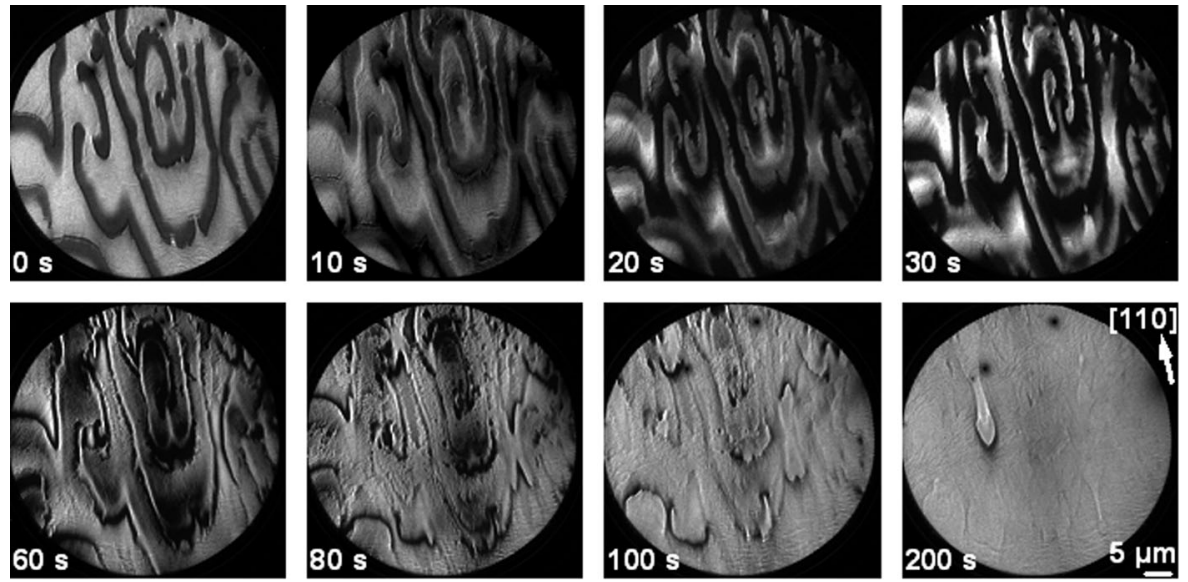

Fig. 8 MEM images showing the effect of in situ evaporation of $0.08 \mathrm{ML}$ potassium on chemical wave patterns starting from a K-free surface (evaporation time $70 \mathrm{~s}$ ). In the upper row of the MEM images $(\mathrm{FOV}=50 \mu \mathrm{m})$ the start voltage was continuously adjusted from $1.0 \mathrm{~V}$ at the beginning to $0.3 \mathrm{~V}$ at the end in order to compensate for the change in electron reflectivity by $\mathrm{K}$ codeposition. For the MEM images in the lower row the start voltage was kept fixed at $0.0 \mathrm{~V}$ and a fixed grey scale was applied. Reaction conditions: $T=468 \mathrm{~K}, p(\mathrm{NO})=2.5 \times 10^{-7} \mathrm{mbar}, p\left(\mathrm{H}_{2}\right)=4.0 \times 10^{-7} \mathrm{mbar}$ (see also movies in ESI, + speed-up factor $=170$ ).

Increasing $p\left(\mathrm{H}_{2}\right)$ to $4.7 \times 10^{-7}$ mbar restores the excitability of the surface and propagating pulses are reestablished again (not shown in Fig. 8). This finding supports the explanation that coadsorbed potassium primarily reduces the $\mathrm{H}_{2}$ sticking coefficient in agreement with the results of earlier studies. ${ }^{26,29}$
Fig. 9 shows the development of a spiral wave pattern during deposition of a large amount of potassium, of $\theta_{\mathrm{K}} \approx 0.12$. As in the preceding experiment, contrast reversal in the imaged chemical pattern occurs between $20 \mathrm{~s}$ and $40 \mathrm{~s}$ deposition time. Similarly to the experiment of Fig. 8, the electron beam energy had to be changed from $0.8 \mathrm{eV}$ used during the first $100 \mathrm{~s}$ (while 

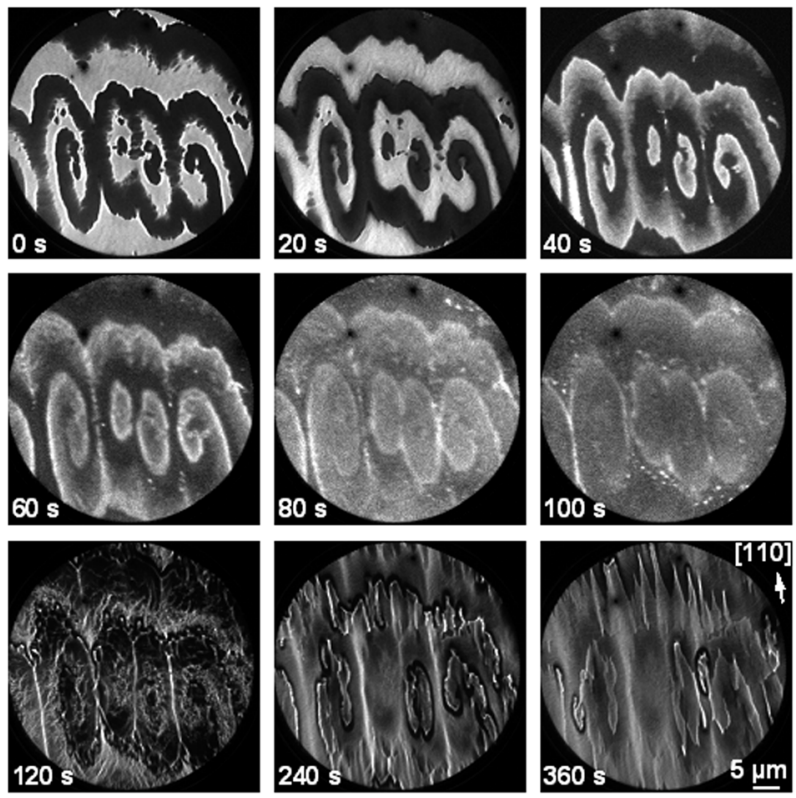

Fig. 9 MEM images showing the decay of wave patterns initiated by in situ evaporation of $0.12 \mathrm{ML}$ potassium starting from a K-free surface (evaporation time $110 \mathrm{~s}$ ). The time when the grey scale of the MEM images started to change was taken as $t=0$ (beginning of K evaporation). FOV of MEM images: $50 \mu \mathrm{m}$. 1st and 2nd row: fixed STV $=0.8 \mathrm{~V}$, adaptive grey scale. 3 rd row: STV $=-0.04 \mathrm{~V}$, fixed grey scale. Reaction conditions: $T=465 \mathrm{~K}, p(\mathrm{NO})=2.5 \times 10^{-7} \mathrm{mbar}, p\left(\mathrm{H}_{2}\right)=9.0 \times$ $10^{-7} \mathrm{mbar}$ (see also movies in ESI, $\dagger$ speed-up factor $=100$ ).

numerically adapting the grey scale) to a value of $0.04 \mathrm{eV}$ afterwards (while applying fixed grey scale imaging). Again the deposition of a large amount of potassium leads finally to a complete suppression of chemical waves but this process involves some intermediate stages. At $t \approx 100 \mathrm{~s}$ the [110]oriented parts of the patterns collide leaving white stripes oriented in the [110]-direction, which persist for several minutes before they vanish. This behavior is reminiscent of the so-called "propagation failure" observed in the $\mathrm{NO}+\mathrm{H}_{2}$ reaction on $\mathrm{Rh}(110) .{ }^{16}$ There, in an excess of $\mathrm{H}_{2}$, the [001]oriented parts of the rectangularly shaped target patterns dissolved leaving only the [110]-oriented parts. Here the behavior is different. The [001]-oriented fronts still propagate along the [110]-direction as finger-like structures until they finally decay and pulse propagation stops. The dominant features of the pattern are white stripes, which develop in areas where neighboring spiral arms oriented along the [110]-direction (their "long direction") collide. These stripes remain visible on a rather long time scale (see images from 120-360 s).

After the chemical waves have been extinguished by deposition of a large $\mathrm{K}$ coverage they can be regenerated by increasing $p\left(\mathrm{H}_{2}\right)$ up to $1.0 \times 10^{-6}$ mbar as demonstrated in Fig. 10. The wave patterns are now irregular and they exhibit a different character than those at low K coverage. The pulses are imaged as dark bands rapidly propagating along the [110]-direction.

Upon collision of these pulses (white arrows in Fig. 10) a dark area forms but the effect is much less pronounced than at low $\mathrm{K}$ coverage. Evidently also here the collision leads to an enrichment of potassium in the collision area but since the
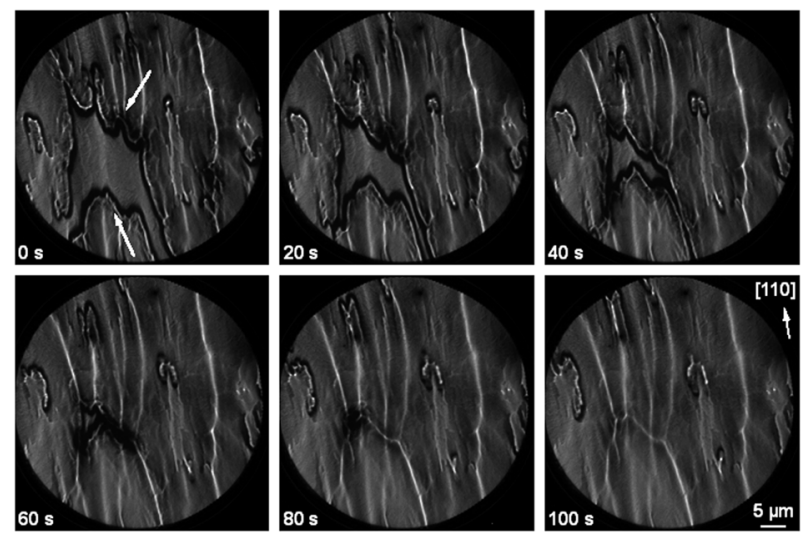

Fig. 10 MEM images showing the formation of almost stationary structures through the collision of pulses on a Rh(110) surface after evaporation of $0.12 \mathrm{ML} \mathrm{K}$ (experiment in Fig. 9). The hydrogen pressure has to be increased relative to the conditions of Fig. 9 in order to reactivate the pattern. MEM: STV $=-0.04 \mathrm{~V}$, fixed grey scale, FOV $=50 \mu \mathrm{m}$. Reaction conditions: $\theta_{\mathrm{K}}=0.12, T=465 \mathrm{~K}, p(\mathrm{NO})=2.5 \times 10^{-7} \mathrm{mbar}, \mathrm{p}\left(\mathrm{H}_{2}\right)=1.01 \times$ $10^{-6} \mathrm{mbar}$ (see also movies in $\mathrm{ESI},+$ speed-up factor $=100$ ).

average $\mathrm{K}$ concentration is already high the relative enrichment is smaller than in Fig. 9. Bright lines perpendicular to the propagating fronts develop upon collision giving the whole pattern the appearance of a cellular structure with the long side of the cells oriented along the [110]-direction. The bright lines forming the network do not move within our temporal and spatial resolution but they are not inert. Upon interaction with pulses, which still evolve they become eroded.

The appearance of the white lines in the MEM images of the cellular patterns can be explained along two different lines. They may either represent a local increase of the WF caused, for example, by chemical changes on the surface or, alternatively, the higher reflectivity may result from a change in the surface morphology. The associated change in the surface potential can cause a lens effect leading to a locally enhanced brightness in the MEM images. The lens effect makes the bright lines difficult to interpret because contrast may also change due to focusing of the microscope.

The white lines in the images cannot be crossed by reduction fronts but are only slowly eroded if fronts propagate along them. Consistent with this inhibition of front propagation would be the development of a stable $\mathrm{K}-\mathrm{O}$ coadsorbate phase with high $\mathrm{K}$ and high oxygen loading. When pulses collide along the [110]direction $\mathrm{K}$ is pushed into an already oxidized surface area what might initiate the formation of such a phase. Since these high coverage $\mathrm{K}+\mathrm{O}$ coadsorbate phases are known to be rather inert with respect to catalytic $\mathrm{H}_{2} \mathrm{O}$ formation, such an explanation would explain the high stability of the white lines. ${ }^{26}$

\section{Discussion}

\subsection{Excitation mechanism with potassium}

The $\mu$ XPS and $\mu$ LEED measurements of this study have demonstrated that the excitation mechanism of the unpromoted system remains basically intact in the presence of coadsorbed potassium. ${ }^{24,25}$ The only significant modification with respect 
to the original mechanism is that the pure oxygen phase, the $\mathrm{c}(2 \times 6)-\mathrm{O}$, has to be replaced as the resting state by a $\mathrm{K}+\mathrm{O}$ coadsorbate phase. The modified excitation cycle comprises the following sequence: $\mathrm{K}+\mathrm{O}$-coadsorbate phase (resting state) $\rightarrow$ $(2 \times 1)-\mathrm{N}$ (excited state) $\rightarrow \mathrm{c}(2 \times 4)-2 \mathrm{O}, \mathrm{N}$ (refractory state) $\rightarrow$ $\mathrm{K}+\mathrm{O}$ coadsorbate phase. In our case the $\mathrm{c}(2 \times 4)$ and the $\mathrm{c}(6 \times 4)$ have been identified as $\mathrm{K}+\mathrm{O}$ coadsorbate phases but it is very likely that under varying reaction conditions and/or $\mathrm{K}$ coverages a different one of the numerous $\mathrm{K}+\mathrm{O}$ phases which exist will occupy this role. ${ }^{20}$ As already pointed out in the Results section, the non-vanishing nitrogen intensity in Fig. 3 is not consistent with this excitation cycle. However, LEEM observations indicate that the $\mathrm{K}+\mathrm{O}$ phase is smaller than our probing window $(>2 \mu \mathrm{m})$. Thus, our micro-probe spectroscopy measurements are sensing the area surrounding the front, which results in a non-zero nitrogen intensity due to the limited spatial resolution.

Alternatively, one could consider an incomplete transition from the refractory to the resting state. Effectively this would lead to an inhomogeneous surface where both phases are separated in coexisting islands on a nm length scale. Both possibilities, a very thin but still macroscopic $(\approx 1 \mu \mathrm{m}) \mathrm{K}+\mathrm{O}$ phase and an inhomogeneous transition with coexisting islands of nm-size would be consistent with the experimental data.

From the reduced reactivity of oxygen in the presence of coadsorbed potassium one should expect that pulses in the promoted system propagate much slower than without potassium. A comparison of the available data reveals that the effect if it exists is in any case not drastic, i.e. the reduction in velocity is definitely far below one order of magnitude. ${ }^{28}$ A look at the titration data of $\mathrm{Rh}(110) / \mathrm{K}+\mathrm{O}$ reveals that the reduction in reactivity against $\mathrm{H}_{2}$ is small at low $\mathrm{K}$ coverages, at $\theta_{\mathrm{K}} \approx 0.05$, only about a factor of two higher $\mathrm{H}_{2}$ exposures are needed with respect to the K-free surface. ${ }^{26}$ Only when the $\mathrm{K}$ coverage is increased to $\theta_{\mathrm{K}} \approx 0.12$ the effect becomes drastic as evidenced by an increase of the $\mathrm{H}_{2}$ exposure required for complete reactive removal by more than one order of magnitude. ${ }^{26}$

Since the $\mathrm{c}(2 \times 4)$-K, $\mathrm{O}$ which has been identified in the $\mu$ LEED measurements exhibits a $\mathrm{K}$ coverage around $\theta_{\mathrm{K}}=0.12$, one expects that the front velocity should in fact be reduced strongly compared to the K-free surface which is obviously not the case. If the $\mathrm{c}(2 \times 4)-\mathrm{K}, \mathrm{O}$ structure is just a minority phase surrounded by a disordered $\mathrm{K}+\mathrm{O}$ phase with a smaller potassium coverage, then the small reduction in front velocity might not be too surprising. Hydrogen might adsorb in other areas with a smaller $\mathrm{K}$ coverage and hence higher $\mathrm{H}_{2}$ sticking coefficient and diffuse from there to $\mathrm{c}(2 \times 4)$ islands to react off the oxygen. We observe in fact that a rising $\mathrm{K}$ coverage slows down pulse propagation: at a too high $\mathrm{K}$ coverage pulse propagation is simply completely suppressed. In between no or only a small effect on pulse speed and complete suppression we see no gradual transition.

\subsection{Mechanism of potassium transport}

The main result of this study with respect to mass transport is that a high potassium concentration builds up in front of the pulse. Apparently, the nitrogen adlayer in a pulse is pushing the
$\mathrm{K}$ atoms whereas the alternative possibility that the oxygen is dragging the potassium has to be discarded. Simulations which only comprised attractive interactions between coadsorbed $\mathrm{K}$ and $\mathrm{O}$ adatoms failed to generate pulses which were transporting potassium. Intuitively, this is easy to understand because on a surface nearly fully covered with oxygen no energetic driving force exists for the $\mathrm{K}$ atoms to move to a different place. A mathematical model which was based on nitrogen acting as a diffusion barrier for potassium could in fact reproduce all essential experimental observations. ${ }^{14}$ In terms of energetic interactions this mechanism means that repulsive interactions have to exist between nitrogen and coadsorbed potassium. So far, only indirect evidence exists for this repulsion the main argument being that atomic nitrogen and potassium favor different types of reconstructions on $\mathrm{Rh}(110)$. Adsorbed nitrogen induces reconstructions of the added row type, i.e. $\mathrm{Rh}-\mathrm{N}-\mathrm{Rh}$ chain form oriented in the [001]-direction whereas potassium favors reconstructions of the missing-row type characterized by a substrate structure in which every $n$th $(n=2,3,4,5)$ of the close-packed [110]-rows is missing. ${ }^{20}$ A second argument is that adsorbed nitrogen provides a diffusion barrier for potassium. Otherwise two colliding pulses would not be able to compress the potassium caught in between the approaching fronts into a small area.

Potassium diffuses very fast on a bare metal surface but with coadsorbed oxygen the diffusivity is reduced by several orders of magnitude. ${ }^{8,10}$ Using the diffusion parameters from ref. 8 one calculates for a temperature of $500 \mathrm{~K}$ a diffusion constant $D_{\mathrm{K}}$ for potassium which is $4.5 \times 10^{-5} \mathrm{~cm}^{2} \mathrm{~s}^{-1}$ for the oxygen free $\mathrm{Rh}(110)$ surface and only $5.2 \times 10^{-9} \mathrm{~cm}^{2} \mathrm{~s}^{-1}$ for a $\mathrm{Rh}(110)$ surface covered with $0.8 \mathrm{ML}$ of oxygen, i.e. a reduction by four orders of magnitude. This reduction in mobility by coadsorbed oxygen has been attributed to the strong chemical attraction between $\mathrm{K}$ and $\mathrm{O}$ which enhances the energetic corrugation of the surface. Starting with reactive removal of oxygen by an advancing reaction front the mass transport of potassium with pulses could proceed in the following way. As the oxygen in the $\mathrm{K}+\mathrm{O}$ coadsorbate is reacted off the potassium atoms which are no longer hold by the strong $\mathrm{K}-\mathrm{O}$ bonds are liberated and can diffuse freely. In the backward direction their diffusion is hindered by the nitrogen adlayer of the pulse which acts as a diffusional barrier. However, in the forward direction, i.e. the direction in which the pulse moves diffusion is not restricted and the oxygen which still is present in front of the initial reaction front even provides a thermodynamic driving force to diffuse in this direction. As soon as the liberated $\mathrm{K}$ atoms, however, enter the still oxygen covered part they will be nearly immobilized by the strong chemical attraction between $\mathrm{K}$ and $\mathrm{O}$. As a consequence a large $\mathrm{K}$ coverage piles up in front of the pulse and this potassium is transported with the pulse. A repulsion between coadsorbed nitrogen and potassium on $\mathrm{Rh}(110)$ can be deduced from the observation that both adsorbates favor different types of reconstructions. ${ }^{19,20}$

Not all of the potassium is transported with a pulse as shown in Fig. 2. A nitrogen adlayer is not able to block completely potassium diffusion which can be either due to structural imperfections in the 
adlayer or due to a relatively weak repulsion between coadsorbed potassium and nitrogen. The same conclusion can also be reached from the in situ potassium evaporation experiments (Fig. 7) which showed that some of the evaporated potassium remains adsorbed within the nitrogen adlayer.

\subsection{Memory effect and complex patterns}

The complex patterns like the "spider web" which were observed in the K-promoted system were characterized by slowly changing features representing some kind of memory effect in the system. ${ }^{15}$ Structural modifications of the substrate or the formation of some less reactive oxide-like compounds are potential candidates for explaining the memory effect. With the reduced total pressure at which these experiments had to be conducted patterns like the "spider web" could not be reproduced but the experiments revealed a possible mechanism to generate less reactive slowly changing elements in a pattern.

The exact nature of the bright lines forming the network in Fig. 10 has not been unambiguously clarified. They (i) exhibit either a higher WF due to chemical changes on the surface or (ii) are caused by morphological changes of the surface. Their appearance in MEM imaging may also result from a lens effect when the surface potential varies strongly within a short distance. Contrast caused by lens effects depends also on the focusing of the microscope; hence the interpretation of contrast in MEM has to be considered with great care. A plausible hypothesis was formulated based on the observations that the white lines exhibit a reduced reactivity and that potassium favors the formation of very stable high coverage $\mathrm{K}+\mathrm{O}$ coadsorbate structures like the $(8 \times 2)$ phase. The latter structure was found in the $\mathrm{O}_{2}+\mathrm{H}_{2}$ reaction on a K-promoted $\mathrm{Rh}(110)$ surface; the $(8 \times 2)$ was shown to be rather inert with respect to the catalytic reaction..$^{20,26}$ If the enhanced $K$ concentration which builds up in the area in between colliding pulses favors the formation of a stable high coverage $\mathrm{K}+\mathrm{O}$ coadsorbate phase structures like the $(8 \times 2)$-K,O could form the "membranes" in a cellular structure. One can speculate whether in addition to the formation of less reactive high coverage phases also some roughening/facetting takes place but the present LEEM experiments provide no experimental evidence for such effects.

\section{Conclusions}

The propagation of pulses in the $\mathrm{NO}+\mathrm{H}_{2}$ reaction on a K-promoted Rh(110) surface has been studied using LEEM/ MEM, $\mu$ XPS, and $\mu$ LEED. It was shown that the excitation cycle of the unpromoted system remains essentially unchanged if one replaces the $\mathrm{c}(2 \times 6)-\mathrm{O}$ as the resting state of the unpromoted system by $\mathrm{K}+\mathrm{O}$ coadsorbate phases of the K-promoted system. In front of the nitrogen adlayer of pulses potassium is enriched so that potassium is transported with the pulses. Evidently the nitrogen adlayer acts as a diffusional barrier for potassium thus pushing the potassium instead of the potassium being dragged by oxygen. Memory effects can arise in the system through the collision of pulses causing the formation of nearly stationary structures with higher WF and reduced reactivity. The less reactive non-moving structures thus created can potentially act as building blocks for complex patterns.

\section{References}

1 Chemical Waves and Patterns, ed. R. Kapral and K. Showalter, Kluwer, Dordrecht, 1994.

2 R. Imbihl and G. Ertl, Chem. Rev., 1995, 95, 697-733.

3 R. Imbihl, in Handbook of Surface Science, ed. E. Hasselbrink and B. Lundquist, Elsevier, Amsterdam, 2008, vol. 3.

4 Y. De Decker, H. Marbach, M. Hinz, S. Günther, M. Kiskinova, A. S. Mikhailov and R. Imbihl, Phys. Rev. Lett., 2004, 92, 198305; Y. De Decker and A. S. Mikhailov, J. Phys. Chem. B, 2004, 108, 14759.

5 S. C. Glotzer, E. A. Di Marzio and M. Muthukumar, Phys. Rev. Lett., 1995, 74, 2034.

6 Y. Tabe and H. Yokoyama, Langmuir, 1995, 11, 4609.

7 V. V. Vanaq and I. R. Epstein, Phys. Chem. Chem. Phys., 2009, 11, 897.

8 M. Hinz, S. Günther, H. Marbach and R. Imbihl, J. Phys. Chem. B, 2004, 108, 14620.

9 M. Kiskinova, Poisoning and Promotion in Catalysis Based on Surface Science Concepts, in Studies in Surface Science and Catalysis 70, ser., ed. B. Delmon and J. T. Yates, Elsevier, Amsterdam, 1992.

10 H. Marbach, S. Günther, B. Luerßen, L. Gregoratti, M. Kiskinova and R. Imbihl, Catal. Lett., 2002, 83, 161.

11 A. Locatelli, C. Sbraccia, S. Heun, S. Baroni and M. Kiskinova, J. Am. Chem. Soc., 2005, 127, 2351.

12 A. Locatelli, T. O. Mentes, L. Aballe, A. Mikhailov and M. Kiskinova, J. Phys. Chem. B, 2006, 110, 19108.

13 H. Marbach, S. Günther, T. Neubrand and R. Imbihl, Chem. Phys. Lett., 2004, 349, 64.

14 L. Hong, H. Uecker, M. Hinz, Q. Liang, I. G. Kevrekidis, S. Günther, A. Locatelli and R. Imbihl, Phys. Rev. E: Stat. Phys., Plasmas, Fluids, Relat. Interdiscip. Top., 2008, 78, 055203(R).

15 L. Hong, M. Hesse and R. Imbihl, J. Phys. Chem. C, 2009, 113, 4174-4183.

16 F. Mertens and R. Imbihl, Nature, 1994, 370, 124; N. Gottschalk, et al., Phys. Rev. Lett., 1994, 73, 3483.

17 A. Makeev, M. Hinz and R. Imbihl, J. Chem. Phys., 2001, 114, 9083.

18 T. Schmidt, A. Schaak, S. Günther, B. Ressel, E. Bauer and R. Imbihl, Chem. Phys. Lett., 2000, 318, 549.

19 G. Comelli, V. R. Dhanak, M. Kiskinova, K. C. Prince and R. Rosei, Surf. Sci. Rep., 1998, 32, 165.

20 S. Günther, R. Hoyer, H. Marbach, R. Imbihl, F. Esch, C. Africh and G. Comelli, J. Chem. Phys., 2006, 124, 014706.

21 Y. Xu, H. Marbach, R. Imbihl, I. G. Kevrekidis and M. Mavrikakis, J. Phys. Chem. C, 2007, 111, 7446.

22 T. Schmidt, S. Heun, J. Slezak, J. Diaz, K. C. Prince, G. Lilienkamp and E. Bauer, Surf. Rev. Lett., 1998, 5, 1287-1296.

23 A. Locatelli, L. Aballe, T. O. Mentes, M. Kiskinova and E. Bauer, Surf. Interface Anal., 2006, 38, 1554-1557. 
24 F. Mertens, S. Schwegmann and R. Imbihl, J. Chem. Phys., 1997, 106, 4319.

25 A. Schaak, E. Schütz, F. Esch, M. Hinz, S. Günther, M. Marsi, M. Kiskinova and R. Imbihl, Phys. Rev. Lett., 1999, 83, 1882. 26 S. Günther, H. Marbach, R. Imbihl, A. Baraldi, S. Lizzit and M. Kiskinova, J. Chem. Phys., 2003, 119, 12503.
27 A. Baraldi, V. R. Dhanak, M. Kiskinova and R. Rosei, Appl. Surf. Sci., 1994, 78, 445.

28 F. Mertens and R. Imbihl, J. Chem. Phys., 1996, 105, 4317.

29 H. Marbach, S. Günther, T. Neubrand, R. Hoyer, L. Gregoratti, M. Kiskinova and R. Imbihl, J. Phys. Chem., 2004, 108, 15182. 